\title{
Chronic Liver Disease and Silymarin: A Biochemical and Clinical Review
}

\author{
Sean P. Tighe ${ }^{1}$, Daud Akhtar ${ }^{2}$, Umair Iqbal*33 and Aijaz Ahmed ${ }^{4}$ \\ ${ }^{1}$ Division of Gastroenterology and Hepatology, Stanford University School of Medicine, Stanford, CA, USA; ${ }^{2}$ Department of \\ Medicine, University of British Columbia, Vancouver, BC, Canada; ${ }^{3}$ Department of Gastroenterology and Hepatology, Geisinger \\ Commonwealth School of Medicine, Danville, PA, USA; ${ }^{4}$ Division of Gastroenterology and Hepatology, Stanford University School \\ of Medicine, Stanford, CA, USA
}

\begin{abstract}
Chronic liver disease (CLD) is an under-recognized epidemic that continues to increase in prevalence and is a major health concern. Silymarin, the active compound of Silybum marianum (Milk thistle), has historically been used in CLD. A significant barrier to silymarin use is its poor bioavailability. Attempts at improving the bioavailability of silymarin have led to a better understanding of formulation methods, pharmacokinetics, dosing, and associated drug interactions. Clinically, silymarin exerts its hepatoprotective effects through antioxidative, antifibrotic, anti-inflammatory, antitoxin, and anticancerous mechanisms of actions. Despite the use of silymarin being extensively studied in alcoholic liver disease, metabolic-associated fatty liver disease, viral hepatitis, and drug-induced liver injury, the overall efficacy of silymarin remains unclear and more research is warranted to better elucidate the role of silymarin in CLD, specifically regarding its anti-inflammatory effects. Here, we review the current biochemical and clinical evidence regarding silymarin in CLD.

Citation of this article: Tighe SP, Akhtar D, Iqbal U, Ahmed A. Chronic liver disease and silymarin: A biochemical and clinical review. J Clin Transl Hepatol 2020;8(4):454-458. doi: 10.14218/JCTH.2020.00012.
\end{abstract}

\section{Introduction}

Over the last decade, there has been a significant increase in the burden of chronic liver disease (CLD) due to the growing prevalence of metabolic-associated fatty liver disease (MAFLD), with CLD now a major cause of morbidity and mortality worldwide. ${ }^{1}$ The increasing numbers of patients at risk for cirrhosis and in need of liver transplantation have become important economic and health concerns, with studies showing hospitalizations due to CLD having doubled in number over the last decade. ${ }^{2,3}$ Therefore, there is a need

Keywords: Silymarin; Chronic liver disease; Silybum marianum; MAFLD; ALD; Pharmacokinetics.

Abbreviations: ALD, alcoholic liver disease; AST, aspartate aminotransferase ALT, alanine aminotransferase; CLD, chronic liver disease; HCC, hepatocellula carcinoma; HCV, hepatitis C virus; MAFLD, metabolic-associated fatty liver disease; RCT, randomized control trials.

Received: 27 February 2020; Revised: 20 May 2020; Accepted: 8 September 2020

*Correspondence to: Umair Iqbal, Department of Gastroenterology and Hepatology, Geisinger Commonwealth School of Medicine, Danville, PA 17821, USA Tel: +1-570-271-6211, E-mail: uiqbal@geisinger.edu for affordable and effective treatment modalities to reduce the morbidity and mortality associated with CLD. ${ }^{4}$ Certain medicinal plants, such as Silybum marianum, more commonly known as milk thistle, have historically been used for the treatment and prevention of liver disorders. Specifically, silymarin has shown promising protective effects in preclinical studies using a number of formulations, including Legalon which contains the Eurosil 85 formulation. ${ }^{5-8}$ The aim of this article is elaborate on the biochemistry of silymarin pertaining to its formulation, pharmacokinetics, dosing, drug interactions, mechanism of action, while also reviewing the current evidence of silymarin use in chronic liver disease.

\section{Biochemistry}

\section{Formulation}

Silymarin is a complex mixture that includes an array of different flavonolignan isomers. Silybin, one of these isomers, composes up to $50 \%$ of the silymarin mixture and plays an important role in the antioxidative effects of silymarin. These antioxidant effects are a result of silybin diastereomers that undergo biotransformation, leading to the formation of glucuronide derivatives (Fig. 1). ${ }^{9}$

A significant barrier to the clinical use of silymarin is its poor bioavailability, due to its lipophilic nature, and subsequent poor solubility. ${ }^{9}$ Attempts at improving the solubility of silymarin formulations has led to the development of many different commercially tested forms of silymarin, which differ in their composition of silybin. Moreover, approximately 75 silymarin brands have been developed in various dosage forms, such as tablets like Carsil, syrups like Alrin-B, and capsules like

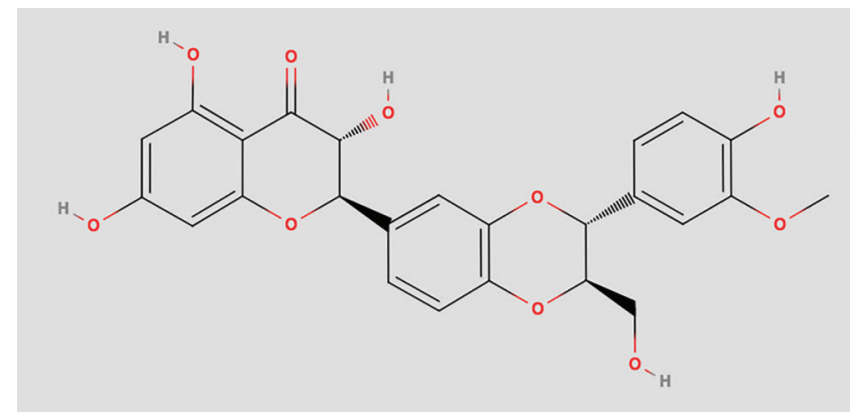

Fig. 1. Chemical structure of silybin. 
Legalon. ${ }^{9}$ Legalon contains Eurosil $85^{\circ}$, which is a standardized preparation method that contains $60 \%$ of silybin and has been used in studies examining the effects of silymarin.

Despite standardized preparation methods, the bioavailability of silymarin can further be affected by genetic polymorphisms and the presence of liver disease. Specifically, the plasma half-life of silybin, the bioactive component in silymarin formulations, is 6 hours, while peak plasma concentrations are usually reached 2-4 hours after administration. Genetic polymorphisms, such as ABCB1 C3435T, however, can affect silybin bioavailability, with one study in healthy patients illustrating varying peak and half times for oral doses of $80 \mathrm{mg}$ of silybin equivalents when compared to plain silymarin capsules. ${ }^{10}$ In addition to genetic polymorphisms, the bioavailability of silymarin can also be significantly affected by the presence of liver disease due to alterations in liver metabolism. Studies have also shown that the effectiveness of silymarin varies between MAFLD and hepatitis C virus (HCV)infected patients. This is a result of higher flavonolignan plasma concentrations and more extensive enterohepatic cycling. ${ }^{11}$

\section{Dosing}

Presently, silymarin is available in a variety of different forms, including capsules and tablets of different strength, with a recommended daily dosage between $420 \mathrm{mg}$ to $600 \mathrm{mg}$. Clinical studies have been conducted with varying doses, ranging as low as $80 \mathrm{mg}$ and as high as $1600 \mathrm{mg}$. One study tested various amounts (160,240, and $360 \mathrm{mg} /$ day) and found statistically significant decreases in liver enzymes in the 240 and $360 \mathrm{mg} /$ day groups in patients with alcoholic liver disease (ALD) and chronic viral hepatitis. ${ }^{12}$ Another study on HCV-decompensated cirrhotic patients concluded that higher doses of silymarin (1.05 $\mathrm{g} /$ day) is superior to a standard dose (420 mg/day). ${ }^{13}$ Although the data from some studies point towards a link between higher concentrations of silymarin and better treatment results and patient outcomes, certain patient populations, such as those with hepatocellular carcinoma (HCC), have not shown the same signal. ${ }^{14}$ Dosage adjustments may, therefore, be necessary to exert a similar effect in patients with liver disease.

\section{Drug interactions}

The drug interaction profile of silymarin has been studied extensively both in laboratory/animal models and clinical trials. ${ }^{15,16}$ Studies with human hepatocytes demonstrate negligible inhibition of CYP450 enzymes at supratherapeutic silymarin concentrations, suggesting that at therapeutic doses silymarin is unlikely to cause hepatocyte related drug-drug interactions. ${ }^{15}$ Silymarin may be indirectly implicated in this reduction, as an additive effect secondary to interactions with antihyperglycemic agents. Furthermore, studies have shown that silymarin can theoretically interfere and effect the clearance of other drugs, such as statins, glucorinidated drugs, and immunosuppressants, such as sirolimus.

\section{Mechanism of action}

The hepatoprotective effects of silymarin are due to its antioxidative, antifibrotic, regenerative, choleretic, immunomodulatory, and anti-inflammatory properties, as illustrated in Fig. 2. ${ }^{17}$

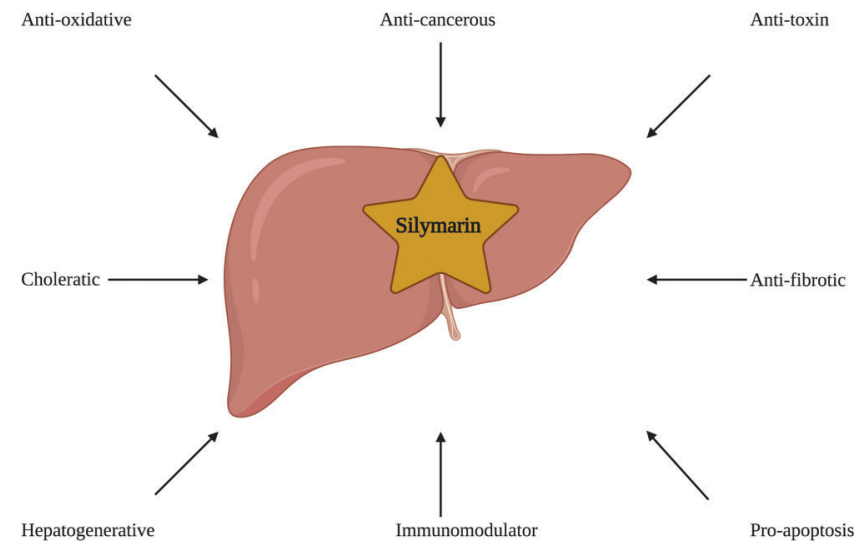

Fig. 2. Hepatoprotective mechanisms of action of silymarin.

\section{Antioxidative effect}

The antioxidant properties of silymarin arise from its ability to utilize scavengers, allowing for the elimination of free radicals. Silymarin's antioxidant activities have different potential mechanisms. These include the inhibition of reactive oxygen speciesproducing enzymes that prevent free radical formation, scavenging of said free radicals, intestinal ion chelation, promoting protective molecule synthesis, and antioxidant enzyme activation. ${ }^{18}$ The antioxidant properties of silymarin have been demonstrated to restore NAD+ homeostasis, sirtuin 1 activity, and the AMP-activated protein kinase $\alpha$ pathway to improve poly(ADP-ribose)-polymerase function (all-important regulatory pathways linked with oxidative stress). ${ }^{19}$ Furthermore, the antioxidant capabilities of silymarin improve the hepatic lipid homeostasis by decreasing de novo lipogenesis via the downregulation of peroxisome proliferator-activated receptor $\gamma_{r}$ acetyl-CoA carboxylase, and fatty acid synthase. ${ }^{19-22}$

\section{Antifibrotic effect}

The antifibrotic activity of silymarin is primarily due to its ability to inhibit the conversion of hepatic stellate cells into myofibroblasts through the inhibition of fibrogenic pathways, such as those implicated in cytoskeletal formation, profibrogenic collagen, and electron transfer chains. Specifically, silymarin down-regulates TGF-B1 mRNA, inhibits NF-kB, and prevents the stimulation of hepatic stellate cells. These findings are supported by studies in animal models, whereby silymarin was shown to slow down the progression of early fibrosis. ${ }^{23,24}$

\section{Anti-inflammatory effect}

The immunomodulatory activity of silymarin exerts an antiinflammatory effect by preventing the activation of the inflammasomes, and NF-кB, which are important in regulating the immune response in inflammatory states. ${ }^{17}$ Silymarin can also restore a pathway known as insulin receptor substrate-1/PI3K/Akt, which can reduce MAFLD-induced insulin resistance and steatosis, as well as activate the farnesyl $X$ receptor, which in turn can diminish hepatic inflammation. 20,25,26 Silymarin's anti-inflammatory and antioxidant capabilities have also been shown to reduce virus-related damage to the liver in chronic HCV infection. ${ }^{27}$ 
Tighe S.P. et al: Silymarin in chronic liver disease

\section{Antitoxin effect}

In cases of drug/toxin-related hepatic injury, the primary mechanisms by which silymarin protects against further damage is through the regulation of membrane permeability and the competitive inhibition of toxins at specific binding sites. This prevents the absorption of these harmful substances, particularly in the hepatic phalloidin-transporting system. ${ }^{28,29}$

\section{Anticancerous effect}

Silymarin also demonstrates anticancerous effects believed to be linked to the inhibition of oxidative stress, promotion of apoptosis, cell cycle arresting, and mitochondrial pathway inhibition. ${ }^{14}$ In vitro and in vivo assays, as well as animal models with HCC treated with silymarin, have showcased the antitumoral effects at varying stages of hepatocarcinogenesis (initiation, promotion, and progression). ${ }^{17}$ Silymarin's ability to aid in hepatic regeneration is also an important characteristic that makes it well suited as a potential therapy in patients with CLD. Specifically, there is an association with ribosomal RNA synthesis, possibly through the stimulation of polymerase I. ${ }^{17}$

\section{Current evidence of silymarin in CLD}

The clinical applications of silymarin encompass a broad range of CLD. These include ALD, MAFLD, drug-and toxininduced liver disease, cholestasis (both pregnancy and nonpregnancy related), primary liver malignancies (including both cholangiocarcinoma and HCC), and viral hepatitis. ${ }^{17}$

\section{$\boldsymbol{A L D}$}

Alcohol is a key risk factor for liver diseases and is responsible for about half of all liver-related cirrhosis. Significant alcohol consumption initially leads to a fatty liver, which can progress to cirrhosis. ${ }^{30}$ The use of silymarin in ALD is limited, due to the poor design of initial studies. A recent systematic review assessing the role of silymarin in patients with ALD suggested that better clinical trials are indicated in order to determine whether or not there is a role for silymarin in ALD management. ${ }^{31}$

\section{MAFLD}

On the contrary, the use of silymarin for MAFLD, appears to better supported with multiple randomized control trials (commonly referred to as RCTs) showing benefit from silymarin use. ${ }^{32}$ MAFLD is a clinical spectrum that can manifest with hepatic fibrosis and inflammation. This is due to the associations between MAFLD and the aforementioned, "anti-inflammatory, antioxidant, antifibrotic, and proregenerative effects of silymarin, in conjunction with its metabolic actions on insulin resistance and hyperlipidemia". ${ }^{17,33,34}$ With a recent meta-analysis involving 587 patients suggesting an improvement in liver function in patients with MAFLD, the use of silymarin for the treatment of MAFLD is promising but still requires further analysis with larger standardized RCTs. ${ }^{32}$

\section{Viral hepatitis}

The advent of antiviral therapy has dramatically changed the landscape of viral hepatitis management. Despite this, high treatment costs and issues with accessibility has created a possible niche for other treatment modalities, such as silymarin. Unfortunately, the efficacy of silymarin when compared to placebo in a well-designed double-blinded trial by Fried et al. ${ }^{35}$ did not significantly reduce serum alanine aminotransferase (ALT) levels. A systematic meta-analysis further demonstrated slightly reduced ALT and aspartate aminotransferase (AST) levels in HCV patients taking silymarin, but these effects were proven to be too variable to provide any concrete clinical significance. ${ }^{36}$

\section{Drug-induced liver injury}

Drug- and toxin-induced liver injury can result from harmful increases in oxidative stress from exposure to various chemicals. ${ }^{37}$ Normally, a balance exists between free radical production and the human body's ability to produce counter-acting antioxidants as a corresponding defense mechanism. However, in drug- and toxin-induced liver injury, the pathogenesis is associated with an imbalance between these two, which explains why silymarin, with its antioxidant effects, is a considerable treatment option for CLDs including jaundice, cirrhosis, and hepatitis. ${ }^{38}$ Studies examining $\mathrm{CCl}_{4}$-hepatotoxicity in rats showed the prevention of hepatic dysfunction and the restoration of normal liver functionality with silymarin use. ${ }^{39,40-44}$ Silymarin aids as an antioxidant, not only by scavenging for free radicals but also by preventing the loss of the antioxidant glutathione. ${ }^{18}$ One study known as Hep573 demonstrated that an intervention of silymarin and antioxidants in a complex naturopathic mixture might lead to normal ALT levels in HCV patients as well as an overall improved quality of life, which includes treating comorbidities that are not always properly treated with the antiviral 'cure'. ${ }^{45}$ These results were mostly found in patients with the specific HCV genotype 1, which can be explained by these individuals lacking endogenous antioxidants, like glutathione. ${ }^{46}$ These findings could be applied to any of the aforementioned liver diseases that result from antioxidant deficiencies and/or free-radical surpluses. Although, various studies have shed a positive light on the beneficial effects of silymarin in different liver diseases, these studies are limited by the volume of data and thus are not adequate for robust conclusions. This, therefore, warrants the need for more quality studies examining silymarin before it can be used as an official clinical treatment in CLD.

\section{Conclusions}

Silymarin is a potent inhibitor of inflammation, fibrosis and oxidative stress that is safe and has low risks of drug interactions. Although some evidence of efficacy exists in a subset of patients with CLD, such as those with ALD and MAFLD, the overall efficacy of silymarin remains unclear, with a recent multiple meta-analysis showing no clinically substantial benefit in the management of CLD. Silymarin may have a role in combination with antioxidants; however, more research is warranted to better elucidate the role of silymarin in the management of CLD. 
Tighe S.P. et al: Silymarin in chronic liver disease

\section{Funding}

None to declare.

\section{Conflict of interest}

The authors have no conflict of interests related to this publication.

\section{Author contributions}

Study concept and design (AA, ST, DA and UI), literature search and drafting of the manuscript (ST, UI and DA), critical revision of manuscript for important intellectual content, senior authorship guidance and supervision (AA). All authors agreed with the final version of the manuscript

\section{References}

[1] Iqbal U, Perumpail BJ, Akhtar D, Kim D, Ahmed A. The epidemiology, risk profiling and diagnostic challenges of nonalcoholic fatty liver disease. Medicines (Basel) 2019;6:41. doi: 10.3390/medicines6010041.

[2] Asrani SK, Hall L, Hagan M, Sharma S, Yeramaneni S, Trotter J, et al. Trends in chronic liver disease-related hospitalizations: A population-based study. Am J Gastroenterol 2019;114:98-106. doi: 10.1038/s41395-018-0365-4.

[3] Kim D, Li AA, Gadiparthi C, Khan MA, Cholankeril G, Glenn JS, Ahmed A. Changing trends in etiology-based annual mortality from chronic liver disease, from 2007 through 2016. Gastroenterology 2018;155:11541163.e3. doi: 10.1053/j.gastro.2018.07.008.

[4] Dennis BB, Akhtar D, Cholankeril G, Kim D, Sanger N, Hillmer A, et al. The impact of chronic liver disease in patients receiving active pharmacological therapy for opioid use disorder: One-year findings from a prospective cohort study. Drug Alcohol Depend 2020;209:107917. doi: 10.1016/j.drugalcdep. 2020.107917.

[5] Song Z, Deaciuc I, Song M, Lee DY, Liu Y, Ji X, et al. Silymarin protects against acute ethanol-induced hepatotoxicity in mice. Alcohol Clin Exp Res 2006;30: 407-413. doi: 10.1111/j.1530-0277.2006.00063.x.

[6] Dehmlow C, Erhard J, de Groot H. Inhibition of Kupffer cell functions as an explanation for the hepatoprotective properties of silibinin. Hepatology 1996;23:749-754. doi: 10.1053/jhep.1996.v23.pm0008666328.

[7] Perumpail BJ, Li AA, Iqbal U, Sallam S, Shah ND, Kwong W, et al. Potentia therapeutic benefits of herbs and supplements in patients with NAFLD. Diseases 2018;6:80. doi: 10.3390/diseases6030080.

[8] Valenzuela A, Garrido A. Biochemical bases of the pharmacological action of the flavonoid silymarin and of its structural isomer silibinin. Biol Res 1994; 27:105-112.

[9] Javed S, Kohli K, Ali M. Reassessing bioavailability of silymarin. Altern Med Rev 2011;16:239-249.

[10] Gatti G, Perucca E. Plasma concentrations of free and conjugated silybin after oral intake of a silybin-phosphatidylcholine complex (silipide) in healthy volunteers. Int J Clin Pharmacol Ther 1994;32:614-617.

[11] Schrieber SJ, Hawke RL, Wen Z, Smith PC, Reddy KR, Wahed AS, et al. Differences in the disposition of silymarin between patients with nonalcoholic fatty liver disease and chronic hepatitis C. Drug Metab Dispos 2011;39:21822190. doi: $10.1124 / d m d .111 .040212$.

[12] Vailati A, Aristia L, Sozze E, Milani F, Inglese V, Galenda P, et al. Randomized open study of the dose-effect relationship of a short course of IdB 1016 in patients with viral or alcoholic hepatitis. Fitoterapia 1993;64:219-231.

[13] Fathalah WF, Abdel Aziz MA, Abou El Soud NH, El Raziky MES. High dose of silymarin in patients with decompensated liver disease: A randomized controlled trial. J Interferon Cytokine Res 2017;37:480-487. doi: 10.1089/jir. 2017.0051.

[14] Mastron JK, Siveen KS, Sethi G, Bishayee A. Silymarin and hepatocellular carcinoma: a systematic, comprehensive, and critical review. Anticancer Drugs 2015;26:475-486. doi: 10.1097/CAD.0000000000000211.

[15] Doehmer J, Tewes B, Klein KU, Gritzko K, Muschick H, Mengs U. Assessment of drug-drug interaction for silymarin. Toxicol In Vitro 2008;22:610-617. doi: 10.1016/j.tiv.2007.11.020.

[16] Fazzi AJ. Natural medicines comprehensive database. J Consum Health Internet 2008;9:79-85. doi: 10.1300/J381v09n02_09.

[17] Abenavoli L, Aviello G, Capasso R, Milic N, Capasso F. Milk thistle for treatment of nonalcoholic fatty liver disease. Hepatitis Monthly 2011;11: 173-177.
[18] Surai PF. Silymarin as a natural antioxidant: An overview of the current evidence and perspectives. Antioxidants (Basel) 2015;4:204-247. doi: 10. 3390/antiox4010204.

[19] Salomone F, Barbagallo I, Godos J, Lembo V, Currenti W, Cinà D, et al. Silibinin restores NAD $\square$ levels and induces the SIRT1/AMPK pathway in nonalcoholic fatty liver. Nutrients 2017;9:1086. doi: 10.3390/nu9101086.

[20] Akhtar DH, Iqbal U, Vazquez-Montesino LM, Dennis BB, Ahmed A. Pathogenesis of insulin resistance and atherogenic dyslipidemia in nonalcoholic fatty liver disease. J Clin Transl Hepatol 2019;7:362-370. doi: 10.14218/JCTH. 2019.00028

[21] Ni X, Wang H. Silymarin attenuated hepatic steatosis through regulation of lipid metabolism and oxidative stress in a mouse model of nonalcoholic fatty liver disease (NAFLD). Am J Transl Res 2016;8:1073-1081.

[22] Cui CX, Deng JN, Yan L, Liu YY, Fan JY, Mu HN, et al. Silibinin Capsules improves high fat diet-induced nonalcoholic fatty liver disease in hamsters through modifying hepatic de novo lipogenesis and fatty acid oxidation. J Ethnopharmacol 2017;208:24-35. doi: 10.1016/j.jep.2017.06.030.

[23] Lieber CS, Leo MA, Cao Q, Ren C, DeCarli LM. Silymarin retards the progression of alcohol-induced hepatic fibrosis in baboons. J Clin Gastroenterol 2003;37:336-339. doi: 10.1097/00004836-200310000-00013.

[24] Trappoliere M, Caligiuri A, Schmid M, Bertolani C, Failli P, Vizzutti F, et al. Silybin, a component of sylimarin, exerts anti-inflammatory and anti-fibrogenic effects on human hepatic stellate cells. J Hepatol 2009;50:1102-1111. doi: 10.1016/j.jhep.2009.02.023.

[25] Zhang Y, Hai J, Cao M, Zhang Y, Pei S, Wang J, et al. Silibinin ameliorates steatosis and insulin resistance during non-alcoholic fatty liver disease development partly through targeting IRS-1/PI3K/Akt pathway. Int Immunopharmacol 2013;17:714-720. doi: 10.1016/j.intimp.2013.08.019.

[26] Gu M, Zhao P, Huang J, Zhao Y, Wang Y, Li Y, et al. Silymarin ameliorates metabolic dysfunction associated with diet-induced obesity via activation of farnesyl X receptor. Front Pharmacol 2016;7:345. doi: 10.3389/fphar.2016. 00345.

[27] Federico A, Dallio M, Loguercio C. Silymarin/silybin and chronic liver disease: A marriage of many years. Molecules 2017;22:191. doi: 10. 3390/molecules22020191.

[28] Serviddio G, Bellanti F, Stanca E, Lunetti P, Blonda M, Tamborra R, et al. Silybin exerts antioxidant effects and induces mitochondrial biogenesis in liver of rat with secondary biliary cirrhosis. Free Radic Biol Med 2014;73: 117-126. doi: 10.1016/j.freeradbiomed.2014.05.002.

[29] Trakulsrichai S, Sriapha C, Tongpoo A, Udomsubpayakul U, Wongvisavakorn $\mathrm{S}$, Srisuma S, et al. Clinical characteristics and outcome of toxicity from Amanita mushroom poisoning. Int J Gen Med 2017;10:395-400. doi: 10. 2147/IJGM.S141111.

[30] Abenavoli L, Masarone M, Federico A, Rosato V, Dallio M, Loguercio C, et al. Alcoholic hepatitis: Pathogenesis, diagnosis and treatment. Rev Recent Clin Trials 2016;11:159-166. doi: 10.2174/1574887111666160724183409.

[31] Ghorbani Z, Hajizadeh M, Hekmatdoost A. Dietary supplementation in patients with alcoholic liver disease: a review on current evidence. Hepatobiliary Pancreat Dis Int 2016;15:348-360. doi: 10.1016/s1499-3872(16) 60096-6.

[32] Zhong S, Fan Y, Yan Q, Fan X, Wu B, Han Y, et al. The therapeutic effect of silymarin in the treatment of nonalcoholic fatty disease: A meta-analysis (PRISMA) of randomized control trials. Medicine (Baltimore) 2017;96: e9061. doi: 10.1097/MD.0000000000009061.

[33] Cacciapuoti F, Scognamiglio A, Palumbo R, Forte R, Cacciapuoti F. Silymarin in non alcoholic fatty liver disease. World J Hepatol 2013;5:109-113. doi: 10.4254/wjh.v5.i3.109.

[34] Milosević N, Milanović M, Abenavoli L, Milić N. Phytotherapy and NAFLD-from goals and challenges to clinical practice. Rev Recent Clin Trials 2014;9:195203. doi: $10.2174 / 1574887109666141216110337$.

[35] Fried MW, Navarro VJ, Afdhal N, Belle SH, Wahed AS, Hawke RL, et al. Effect of silymarin (milk thistle) on liver disease in patients with chronic hepatitis $C$ unsuccessfully treated with interferon therapy: a randomized controlled trial. JAMA 2012;308:274-282. doi: 10.1001/jama.2012.8265.

[36] de Avelar CR, Pereira EM, de Farias Costa PR, de Jesus RP, de Oliveira LPM. Effect of silymarin on biochemical indicators in patients with liver disease: Systematic review with meta-analysis. World J Gastroenterol 2017;23: 5004-5017. doi: 10.3748/wjg.v23.i27.5004.

[37] Williams R. Global challenges in liver disease. Hepatology 2006;44:521526. doi: 10.1002/hep.21347.

[38] Javed S, Ahsan W, Kohli K. Pharmacological influences of natural products as bioenhancers of silymarin against carbon tetrachloride-induced hepatotoxicity in rats. Clin Phytosci 2018;4:18. doi: 10.1186/s40816-018-0079-6.

[39] Gu HR, Park SC, Choi SJ, Lee JC, Kim YC, Han C], et al. Combined treatment with silibinin and either sorafenib or gefitinib enhances their growth-inhibiting effects in hepatocellular carcinoma cells. Clin Mol Hepatol 2015;21: 49-59. doi: $10.3350 / \mathrm{cmh} .2015 .21 .1 .49$.

[40] Wafay H, El-Saeed G, El-Toukhy S, Youness E, Ellaithy N, Agaibi M, et al. Potential effect of garlic oil and silymarin on carbon tetrachloride-induced liver injury. Aust J Basic Appl Sci 2012;6:409-414. 
[41] Tsai JH, Liu JY, Wu TT, Ho PC, Huang CY, Shyu JC, et al. Effects of silymarin on the resolution of liver fibrosis induced by carbon tetrachloride in rats. J Viral Hepat 2008;15:508-514. doi: 10.1111/j.1365-2893.2008.00971.x.

[42] Abrol S, Trehan A, Katare OP. Comparative study of different silymarin formulations: formulation, characterisation and in vitro/in vivo evaluation. Curr Drug Deliv 2005;2:45-51. doi: 10.2174/1567201052772870.

[43] El-Samaligy MS, Afifi NN, Mahmoud EA. Evaluation of hybrid liposomesencapsulated silymarin regarding physical stability and in vivo performance. Int J Pharm 2006;319:121-129. doi: 10.1016/j.ijpharm.2006.04.023.

[44] Yadav NP, Pal A, Shanker K, Bawankule DU, Gupta AK, Darokar MP, et al. Synergistic effect of silymarin and standardized extract of Phyllanthus amarus against $\mathrm{CCl} 4$-induced hepatotoxicity in Rattus norvegicus. Phytomedicine 2008;15:1053-1061. doi: 10.1016/j.phymed.2008.08.002.

[45] Salmond SJ, George J, Strasser SI, Byth K, Rawlinson B, Mori TA, et al. Hep573 study: A randomised, double-blind, placebocontrolled trial of silymarin alone and combined with antioxidants to improve liver function and quality of life in people with chronic hepatitis C. Aust J Herb Naturop Med 2018;30:12-24.

[46] Barbaro G, Di Lorenzo G, Soldini M, Parrotto S, Bellomo G, Belloni G, et al. Hepatic glutathione deficiency in chronic hepatitis $C$ : quantitative evaluation in patients who are HIV positive and HIV negative and correlations with plasmatic and lymphocytic concentrations and with the activity of the liver disease. Am J Gastroenterol 1996;91:2569-2573. 